\title{
Distributed Signal Processing for Dense 5G IoT Platforms: Networking, Synchronization, Interference Detection and Radio Sensing
}

\author{
Gloria Soatti ${ }^{\mathrm{a}}$, Stefano Savazzi ${ }^{\mathrm{b}, *}$, Monica Nicoli $^{\mathrm{c}}$, Maria Antonieta Alvarez ${ }^{\mathrm{a}, \mathrm{d}}$, \\ Sanaz Kianoush $^{\mathrm{b}}$, Vittorio Rampa ${ }^{\mathrm{b}}$, Umberto Spagnolini ${ }^{\mathrm{a}}$ \\ ${ }^{a}$ Politecnico di Milano, DEIB, P.zza L.da Vinci 32, 20133, Milano, Italy \\ ${ }^{b}$ Consiglio Nazionale delle Ricerche (CNR), IEIIT inst., P.zza L. da Vinci 32, 20133, Milano, Italy \\ ${ }^{c}$ Politecnico di Milano, DIG, P.zza L.da Vinci 32, 20133, Milano, Italy \\ ${ }^{d}$ Escuela Superior Politecnica del Litoral (ESPOL), Guayaquil, Ecuador
}

\begin{abstract}
Driven by the fourth industrial revolution (Industry 4.0), future and emerging Internet of Things (IoT) technologies will be required to support unprecedented services and demanding applications for massive machine-type connectivity, with low latency, high reliability and distributed information processing capability. In this article, distributed signal processing methodologies are highlighted as enablers for next generation cloud-assisted IoT systems. The proposed distributed algorithms run inside a wireless cloud network (WCN) platform and are exploited for WCN self-organization, distributed synchronization, networking and sensing. The WCN can lease augmented communication and sensing services to off-theshelf industrial wireless devices via a dense, self-organizing "cloud" of wireless nodes. The paper introduces, at first, the WCN architecture and illustrates an experimental case study inside a pilot industrial plant. Next, it proposes a redesign of consensus-based algorithms for enabling a selected set of distributed information processing functionalities within the WCN platform, with application in practical IoT scenarios. In particular, cooperative communication algorithms are adopted to support reliable communication services. Distributed timing and carrier frequency offset estimation methods are investigated to enable low-latency services through accurate synchronization. Autonomous identification of recurring interference patterns is proposed for multiple access coordination
\end{abstract}

\footnotetext{
${ }^{*}$ Corresponding author (stefano.savazzi@ ieiit.cnr.it)
} 
in the shared 5G spectrum. Finally, localization and vision applications based on distributed processing of wireless signals are investigated to support contact-free human-machine interfaces.

\section{Introduction}

Next generation Internet of Things (IoT) is expected to be underpinned by $5 \mathrm{G}$ wireless communication technologies. Considering the exponentially increasing number of IoT devices [1], in the near future wireless IoT networks will become topologically dense, with huge numbers of complex interactions taking place and evolving towards self-organized architectures. For example, current industrial automation trends towards Industry 4.0 paradigms are driving the transformation of factories into highly flexible and reconfigurable production systems. In this context, radio technologies will play a crucial role only if paired with advanced solutions to support massive machine-type communications (mMTC), ultra-reliable low-latency (URLL) data publishing, as well as decentralized computing capabilities for critical applications demanding for self-organization. Commercial-offthe-shelf (COTS) IoT technologies designed for industrial setups [2, 3], namely Industrial Internet of Things (IIoT), support long-term deployments while communication protocols are primarily designed to maximize battery lifetime $[4,5]$ or optimized to handle periodic or non-critical traffic [6]. To stimulate a wider adoption of wireless networks in an industrial context, substantial technology innovation is thus required in terms of new types of devices embedding a large set of functions in a decentralized mode. Such functions include self-configuring and learning protocols, communication and cloud-assisted computing strategies to support delay/safety-critical applications.

In this paper, we propose distributed signal and information processing techniques applied to a dense cooperative wireless cloud network (WCN) platform. As shown in Fig. 1, the WCN enables augmented communication and sensing services that can be transparently provided to COTS devices via a dense, selforganizing "cloud" of wireless nodes. In this cloud, information is forwarded via multiple relays to the intended destinations using cooperative communications and distributed signal processing tools. Network organization, synchronization and management (i.e., for multiple access to a shared interference-limited spectrum), as well as sensing tools are fully decentralized. COTS devices at the edge are blind to the inner behavior of the cloud, they can access to cloud services through cloud access nodes while the cloud is able to self-organize, providing augmented services on-demand. 
The WCN concept goes beyond theory, in fact it has been developed and demonstrated by focusing on several industry-scale applications [7, 8]. To support WCN functions, distributed signal processing techniques are herein proposed to let the nodes acquire network-state information to set up the cloud functionality and self-organize without the support of any central coordinator. In fact, centralized algorithms require each node to broadcast raw data to a fusion center (FC), which is responsible for processing and sending back the output to all nodes. Although this guarantees optimal performances, these solutions are penalized by the latency and the communication/computational overhead required for data aggregation/processing at the FC, that badly scale with the network size. Furthermore, they are vulnerable to device failure at the FC or closely located nodes. On the contrary, distributed algorithms enable the devices to fuse their sensed data and infer the desired information relying solely on local processing and interactions with neighbors. Such interactions can be exploited to infer, or learn patterns of interest that are hidden in the data sparsely observed by the devices (or agents), make collective decisions, and reveal relationships or recognize behaviors of interest. Even if each agent may not be capable of sophisticated behaviors on its own, the combined action of all agents allows to solve complex tasks [24-33, 35].

The paper is organized as follows: first, in Sect. 2 we provide an overview of the WCN architecture, as well as a description of the reference scenario and the supported cloud functions, ranging from cooperative networking to distributed synchronization and sensing. In line with the $5 \mathrm{G}$ evolution, a multi-RAT (Radio Access Technology) architecture is discussed for critical process monitoring [10], where the WCN nodes employ an IIoT wireless standard to interface with the preexisting field equipment. Cooperative networking functions are adopted in Sect. 3 to set up augmented intra-cloud communications and enable the implementation of distributed processing functions. In Sect. 4, we summarize the theoretical underpinnings of the distributed information processing functions supported by the WCN. In particular, inference, learning, and decision tasks are solved by means of sophisticated methodologies that are the result of a re-design of signal processing architectures and algorithms for integration within the WCN platform [13-15]. These consensus methods combined with cooperative networking functions lay the groundwork for the implementation of advanced services. In Sects. 5 and 6, we evaluate the proposed algorithms for distributed synchronization and for autonomous identification of recurring interference patterns, respectively. In Sect. 7, device-free radio sensing of the environment through radio frequency (RF) signal inspection is discussed. Finally, in Sect. 8, we draw some conclusions and summarize open issues and future developments. 
Ultra-reliable and Low-latency Cloud-assisted IoT

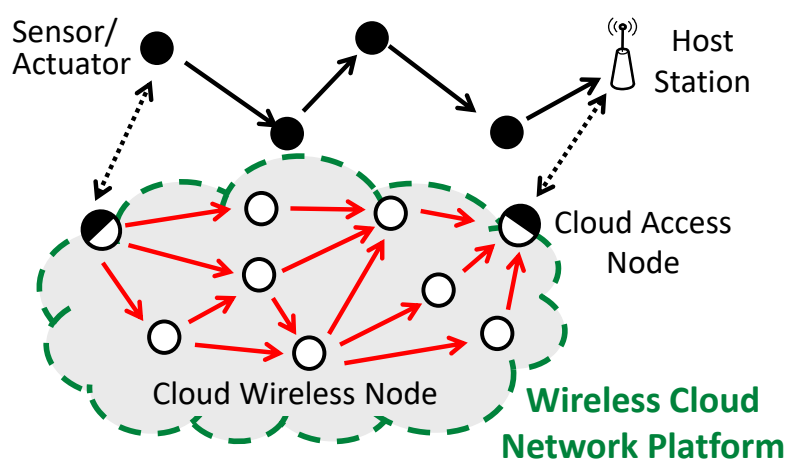

Figure 1: Cloud-assisted IIoT architecture underlaid with a distributed and self-contained wireless cloud network platform.

\section{The WCN Architecture for IIoT}

The WCN platform consists of wireless field devices underlaid with a distributed and self-contained network of cloud nodes (CNs) that can lease advanced networking functions to standard devices upon request. Here, an industry compliant set-up is considered where the field devices comply with the WirelessHART [4] standard (IEC 62591). Cloud devices autonomously self-organize to meet specific service requirements not supported by existing industrial systems. In particular, the proposed WCN design points towards a disruption of the "host-centric" structure of current IoT standards in favor of a "device-centric" architecture that transfers intelligence inside the field device, to support distributed services on demand. Focusing on the industrial framework, this proposal is also in line with the current trend of network level virtualization [16], where the peculiar characteristics of wireless communications pose additional requests, which cannot be easily solved using legacy technologies.

Fig. 2 illustrates the prototype devices of the CNs as well as the PHY/MAC layer interface for device-to-device connectivity. Cloud radio modules are equipped with dual RAT technology. The first radio guarantees the backward compatibility with the existing WirelessHART air-interface, as well as device authentication with the Host station, which controls the industrial monitoring functions [10]. The second radio supports the new cloud functions and adopts a proprietary radio interface. Cloud access (CA) nodes are special Gateway devices that provide an interface to industrial field equipment requesting cloud services and seamless traf- 


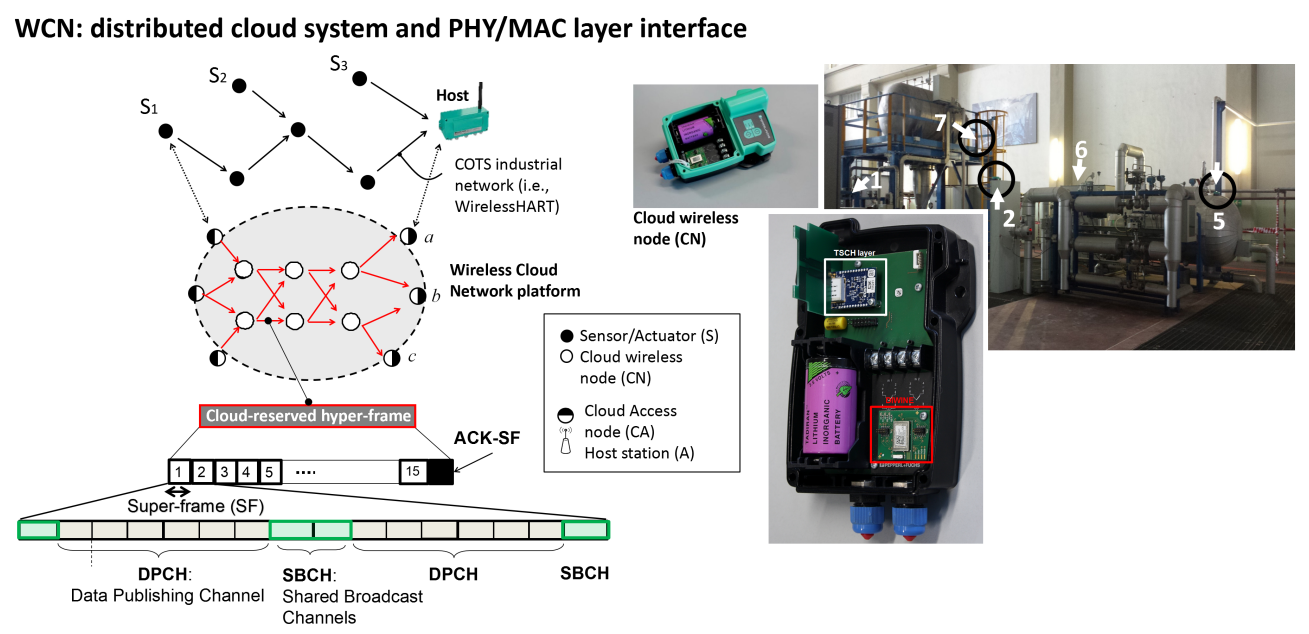

Figure 2: WCN architecture and PHY/MAC interface: dual RAT implementation with batterypowered CN prototypes (courtesy of Pepperl+Fuchs).

fic off-loading. Communication among CA nodes is implemented by cooperative transmissions.

As depicted in the Fig. 2 (at bottom) the communication among the CNs inside the cloud network takes place over a series of contiguous, synchronized slots of $10 \mathrm{~ms}$ each, organized in super-frames of 8 slots and hyper-frames collecting a full communication session of 16 consecutive super-frames. Every $\mathrm{CN}$ has a shared notion of timing and frequency offsets that can be initially provided by the Host station and then maintained periodically by a re-synchronization algorithm. During every super-frame, publishing, or transmission, of data among CNs is implemented over 6 contiguous data publishing channels (DPCH). Two shared broadcast channels $(\mathrm{SBCH})$ are also configured inside each super-frame to propagate $\mathrm{CA}$ control/configuration functions as well as to perform distributed sensing tasks and synchronization functions.

\subsection{Dual Radio Architecture for Implementation}

The $\mathrm{CN}$ is implemented by a dual-processor that handles the dual-radio devices and the related RAT interfaces. One radio is dedicated to the support of cloud functions, namely, networking, synchronization and sensing, while the second one ensures the compatibility with an IEEE 802.15.4 compliant industrial network. Here, the WirelessHART protocol is adopted as the current de-facto solution for industrial process-control applications, also influencing IEEE 802.15.4e 
standardization [11]. The usage of two independent RF radios is crucial to comply with the WCN architecture designed to work in parallel with an existing industrial network, to augment its basic networking and sensing functions. Parallel network operation is implemented by first identifying the channels/bands unused by the industrial network (by spectrum sensing). Then, these resources are assigned to the WCN, so that it could operate simultaneously when needed.

The dual-layer model enables the coexistence of IEEE 802.15.4 based protocol stacks and the "cloud-oriented" layer that integrates the cloud functions. Notice that, in an industrial environment, the product lifetime is usually considerably long for CAPEX reasons. Therefore, any 5G-compliant solution should be designed not only to augment/extend the functionality of the system, but also to guarantee compatibility with existing field-deployed products.

\subsection{Supported Cloud Functions: Networking and Sensing}

Current industrial wireless solutions [2] support few critical applications, with limited scheduling options, due to an optimized design for energy consumption and deterministic traffic management. In addition, unlike wired solutions (i.e., CAN, Fieldbus), the radio link quality is typically impaired by harsh propagation conditions. Building blockage, metallic obstructions, and co-channel interference often impose a certification of the communication reliability through network planning optimization tools as well as post-layout verification [9]. In the following sections, we detail the supported advanced WCN functions to enable relevant mission-critical workloads required in specific industrial applications.

1. Cooperative networking functions. In Sect. 3, we address the cooperative networking functions of the WCN platform to support critical data publishing. Data publishing happens when a field device detects some relevant conditions that generally require either a low-latency reaction, or a highly reliable, or a high-throughput data transfer. In the example of Fig. 3-(b), a low-latency data publishing task is leased to the WCN: the CA nodes handle asynchronous events taking place either at a remote wireless field device or at the Host station (or both) so that suitable corrective actions can be applied. The availability of a low-latency "upstream" (i.e., field device to Host) and "downstream" (i.e., Host to field device) data forwarding mechanism also enables the fast exchange of request-response messages, typically consisting of few datagrams. Validation of fast request-response transactions is also addressed in the same Sect. 3.

2. Distributed signal and information processing functions. Processing tasks through industrial wireless networks are typically performed centrally by 
the Host station, either by applying decisions (i.e., synchronization, multiple access scheduling) based on local procedures, or by collecting observations from all field devices. In Sect. 4- 7, the use of the WCN platform is investigated to support decentralized estimation/monitoring functions. All these functionalities rely on consensus-based decision making policies, as discussed in Sect. 4. Distributed processing techniques enable the CNs to implement complex functions in a fully distributed way, without the support of any central coordinator. A distributed synchronization policy lets the CNs to autonomously (with respect to the Host station) synchronize to a common/shared beacon signature. Distributed synchronization methods and tools are discussed in Sect. 5. They enable the implementation of several decentralized processing functions, and particularly the following ones:

(a) Spectrum sensing: to address (in Sect. 6) the self-learning of interference patterns in the time-frequency (TF) domain. This, in turn, can be used by the WCN to identify the unused portion of the spectrum and to optimally schedule the transmission resources.

(b) Radio sensing for localization and vision: to develop (in Sect. 7) body motion sensing capabilities derived from the WCN platform by using distributed signal processing functionalities. Motion sensing is based on the real-time analysis of radio signals that encode a view of all moving/fixed objects traversed by their propagation [12]. Such techniques are "device-free" as they do not require dedicated radio tags, which are unfit in most industrial environments. Radio sensing is also considered an enabling technology for the development of contact-free human-machine interfaces.

\section{Cooperative Networking for Low-Latency Data Publishing}

In this section, we focus on data publishing functions for throughput- and latency-critical end-to-end communication among CA nodes. As depicted in Fig. 3(a), a sequence of cooperative transmissions is implemented to connect a source and a destination CA node, while the CNs act as intermediate decode and forward relays [17]. The cooperative link abstraction consists here of separate radios encoding/transmitting or decoding/receiving messages in coordination [18, 19]. Experimental validations in controlled laboratory environments (e.g., see [20]) showed that such systems could achieve enhanced reliability compared to standard multi-hop solutions thus mimicking the performance of a wired system. Despite 


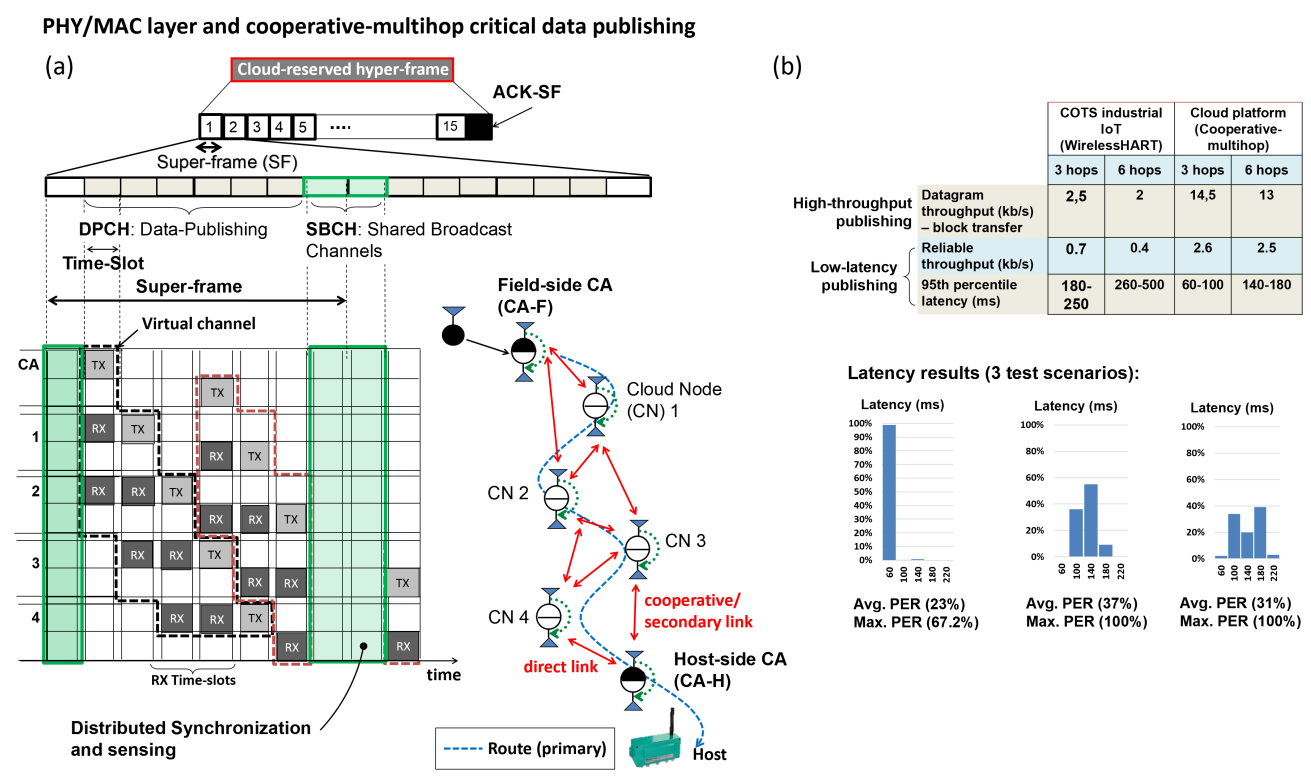

Figure 3: (a): Cooperative multi-hop message passing, chain-start, chain-end and ACK superframes. (b) Latency and throughput comparisons with COTS IIoT designs: a standard WirelessHART network (IEC 62591) is used as benchmark.

some recent attempts to develop cooperative relaying features tailored to wireless IoT networks [19, 21], practical methods to integrate such schemes into an industrial standard are still missing [10]. In the proposed setup, validated in the next section by experiments, the communication among the CNs takes place over consecutive DPCH slots. Within each slot, a single cloud node can thus transmit, while the selected CNs along the route path can receive in "multicast" mode. The cooperative algorithm allows an "implicit-retry" capability resulting from its multiple packet propagation and receive opportunities. In more details, the CA source node tunnels the input datagrams through the cloud section: each $\mathrm{CN}$ here handles data forwarding by "duo-cast" mode (e.g., transmitting the same datagram/packet towards the two following nodes in the route path), until reaching the CA destination node. Thus, each packet always has two propagation opportunities; likewise, the destination node has always two packet receive opportunities, corresponding to a "cooperative diversity" order [19] equal to 2 . In what follows, the performance indicator adopted for performance assessment is the latency (e.g., 95-th percentile), measured with respect to the first successful datagram reception. 


\subsection{Experimental Validation}

In Fig. 3-(b) (on the right), latency and throughput results are discussed by collecting measurements obtained in different sites within a testing industrial plant described in [10]. For all cases, the proposed distributed system handles the delivery of data acting as a "hardware as a service" (HaaS) provider and implementing the cooperative forwarding scheme previously described. The goal is to experimentally verify (i.e., in a real-world test-bed) the performance of data publishing, that is specifically tailored as a complement to regular IIoT designs. The PHY layer of the CN transceiver complies with the IEEE 802.15.4e [4] and operates over the $2.4 \mathrm{GHz}$ band. However, it is configured to double the data-rate $(500 \mathrm{~kb} / \mathrm{s})$ to ensure a substantial publishing rate increase. Reduced noise immunity level (due to scaling down of the direct-sequence spread spectrum - DSSS factor) is counter-balanced by the cooperative transmission chain, as well as by the adoption of a slow frequency hopping policy [10]. System validation shows substantial improvements compared with standard single-path source routing (IEC 62591 compliant) solutions. An order of magnitude increase of throughput was made possible by the cloud (in the range 2-4 Kbyte/s), while a twofold increase in packet delivery rate has been observed in most of the investigated settings. The table of Fig. 3-(b) provides a summary of the achievable figures, focusing on high-throughput datagram transfer $(\mathrm{kb} / \mathrm{s})$, reliable throughput for request-response messages (kb/s) and corresponding 95-th percentile latency (ms). For each case, the cloud platform performance is compared with current COTS implementation (WirelessHART), for typical 3 to 6-hop topologies.

The use of the cooperative multi-hop transmission chain provides a sufficiently high level of immunity to multi-path fading and interference: it thus guarantees high reliability and a reasonable level of communication determinism, being this a crucial requirement for real-time control applications. As depicted in the corresponding histograms shown in Fig. 3-(b), the 95-th percentile latency is below 180 $\mathrm{ms}$ in all cases, considering relatively short (3 hops) to long hopping sequences (6 hops). Notice that, for proper actuation/configuration actions, the desirable communication latency should be $250 \mathrm{~ms}$ or below [10]. The maximum publishing latency can be scaled down from 6 up to 10 times compared to current industrial solutions.

\subsection{Considerations on Energy and Spectrum Efficiency}

All devices (CAs and CNs) deployed for the tests previously described are battery powered (see the $\mathrm{CN}$ prototype in Fig. 2). In our scenarios, the availability of 
20 Ah battery has been assumed and, according to available data, the expected battery life is primarily ruled by the more energy-hungry CA functions. Clearly, the cloud network lifetime depends on how frequent the use of the cloud is solicited by the industrial network to handle critical tasks.

According to the experimental results, the cloud node transceiver in the "activestate" consumes roughly $13 \mathrm{~mA}$ (transmit or receive mode). Likewise, for the core processor, an energy consumption of about $5 \mathrm{~mA}$ at $8 \mathrm{MHz}$ applies. However, the processor does support many low-power operating modes while the average energy consumption will much depend on the programmed duty cycle: in fact, the developed cloud algorithms can be either throughput driven, considering the networking functions, or event-driven, considering the distributed consensus tools (Sect. 4). As far as the core processor is concerned, some non-negligible baseline energy consumption is observed. This is required to support the core MAC layer operations, including distributed consensus-based estimation and spectrum sensing functions. It could be roughly estimated to be in the range 100-500 $\mu \mathrm{A}$. However, distributed synchronization, validated in Sect. 5 on more flexible softwaredefined radio hardware, would require advanced PHY layer operations resulting in larger consumption.

Energy consumption tests showed that, as far as the networking functions are concerned, the implementation of the WCN can reduce the consumption of the existing off-the-shelf WirelessHART network as managing off-loaded traffic/services. Clearly, this comes in exchange for additional cloud devices to be installed.

As regards spectrum usage, the $\mathrm{WCN}$ operates over the crowded $2.4 \mathrm{GHz}$ band that is impaired by co-channel interference originated from the shared use of the spectrum (e.g., WiFi, IPv6/Thread, ZigBee, Bluetooth LE/mesh). Distributed spectrum sensing (Sect. 6) is thus applied and validated to estimate the unused portion of bandwidth where to deploy and implement the WCN functions.

\section{Distributed Signal and Information Processing}

Distributed techniques have been used for solving inference problems in various IoT contexts. Typical applications are environmental monitoring [22], industrial control [10], vehicle positioning and traffic monitoring for intelligent transportation systems [23, 24], network synchronization [25-27], and localization for self-organizing IoT networks [28-31], as well as spectrum sensing [32-34] and resource scheduling [35]. Inference is accomplished by incremental algorithms [36], diffusion strategies [37], alternating direction methods of multipliers 


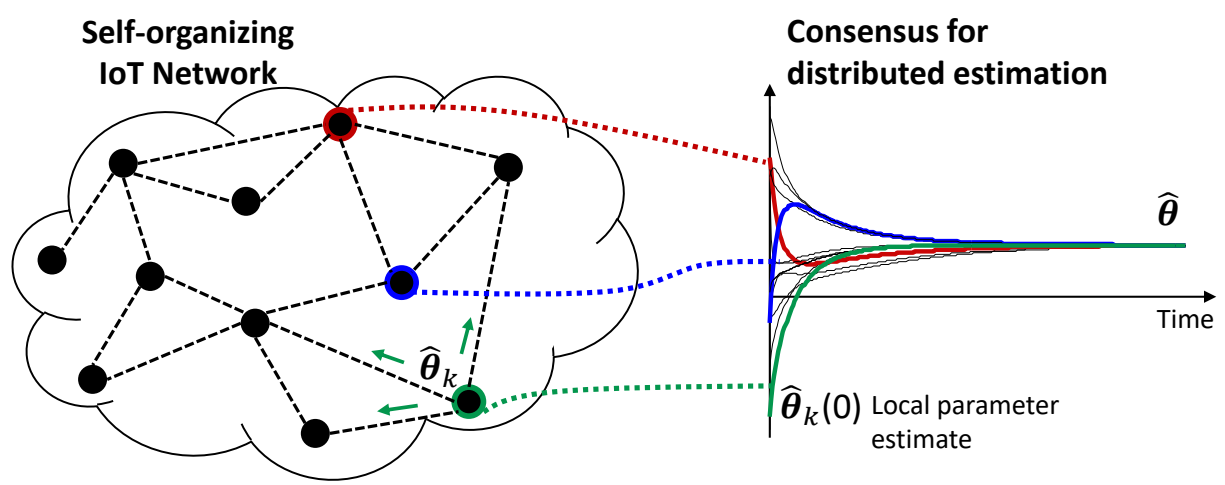

(a)

(b)

Figure 4: (a) Self-organizing IoT network; (b) Example of distributed consensus for the estimation of parameters $\boldsymbol{\theta}$.

(ADMM) [38], belief propagation (BP) [39] or consensus methods [28, 40, 41].

We focus here on consensus methods where estimation is obtained iteratively by successive refinements of local estimates maintained at individual nodes. In the following parts, we briefly review the consensus-based methodology and then, in next sections, we present the implementation within the WCN of Fig. 2 for the solution of specific IoT problems. The analysis shows that this methodology can be designed to closely attain the centralized inference performance, with minimal information exchange and computational burden at each node, adapting also to dynamic conditions with node or link birth/death (e.g., due to device failure).

Let us consider a set of $K \mathrm{CNs}$, distributed over a given area, as depicted in Fig. 4-(a), which have to cooperatively estimate $p$ unknown real-valued parameters, $\boldsymbol{\theta}=\left[\theta_{1} \cdots \theta_{p}\right]^{\mathrm{T}}$. In consensus-based algorithms, the estimate of the parameters of interest $\hat{\boldsymbol{\theta}}$ is computed at each node $k$, with $k=1, \ldots, K$, by successive refinements of the local estimate $\hat{\boldsymbol{\theta}}_{k}(q)$ based on data exchange with neighbors through iterations $q=1,2, \ldots$, until a consensus is reached within the network, i.e., $\hat{\boldsymbol{\theta}}_{k}(\infty) \rightarrow \hat{\boldsymbol{\theta}}$, as shown in Fig. 4-(b). A weighted-consensus approach is considered, where the estimate at node $k$ is updated at iteration $q$ as [28]:

$$
\hat{\boldsymbol{\theta}}_{k}(q+1)=\hat{\boldsymbol{\theta}}_{k}(q)+\varepsilon \mathbf{W}_{k} \sum_{i \in \mathcal{N}_{k}}\left(\hat{\boldsymbol{\theta}}_{i}(q)-\hat{\boldsymbol{\theta}}_{k}(q)\right),
$$

being $\mathcal{N}_{k}$ the set of neighbors for node $k, \mathbf{W}_{k}$ a positive-definite weighting matrix and $\varepsilon$ a step-size parameter. The estimate (1) is known to converge to the weighted average $\hat{\boldsymbol{\theta}}_{k}(\infty)=\left(\sum_{j=1}^{K} \mathbf{W}_{j}^{-1}\right)^{-1} \sum_{k=1}^{K} \mathbf{W}_{k}^{-1} \hat{\boldsymbol{\theta}}_{k}(0)$ of the initial estimates, pro- 
vided that $\varepsilon$ is selected to guarantee convergence [28]. In the conventional average consensus method [40], the weighting matrix is set, $\forall k$, to $\mathbf{W}_{k}=\mathbf{I}_{p}$, with $\mathbf{I}_{p}$ being the $p$-dimensional identity matrix. In this case, the estimate converges to the arithmetic average of the local estimates, $\hat{\boldsymbol{\theta}}_{k}(\infty)=\frac{1}{K} \sum_{k=1}^{K} \hat{\boldsymbol{\theta}}_{k}(0)$. On the other hand, if the weighting matrix is selected as $\mathbf{W}_{k}=\Gamma \mathbf{C}_{k}$, being $\mathbf{C}_{k}$ the covariance of $\hat{\boldsymbol{\theta}}_{k}(0)$ and $\boldsymbol{\Gamma}$ a scaling matrix, the estimate convergences to the optimal (in the minimum-variance sense) centralized solution $\hat{\boldsymbol{\theta}}_{k}(\infty)=$ $\left(\sum_{j=1}^{K} \mathbf{C}_{j}^{-1}\right)^{-1} \sum_{k=1}^{K} \mathbf{C}_{k}^{-1} \hat{\boldsymbol{\theta}}_{k}(0)$, with minimal inter-node signaling (see [28] for details). Note that the conventional approach is sub-optimal with respect to the weighted consensus method as it does not account for the different accuracy of the local estimates at different nodes.

Consensus methodologies are used in the following sections as basis to develop distributed algorithms for self-organization of WCN. Network synchronization (Sect. 5), spectrum sensing (Sect. 6), as well as passive radio sensing (Sect. 7) are considered as special cases.

\section{Distributed Synchronization}

Distributed synchronization is a consensus-type method tailored for dense cloud networks to let the CNs to asymptotically reach a global convergence jointly on time-frequency offset. According to the WCN setting introduced in Sect. 2, each $\mathrm{CN}$ node is equipped with oscillators that run autonomously. Although timefrequency offset compensation can be initially triggered by the Host station (at the WCN formation), a periodic re-synchronization is needed to keep carrier frequency and timing mutually locked among all CNs to enable the WCN functionality. The method is herein based on the exchange of a common beacon signature (i.e., the same beacon is assigned to all $\mathrm{CNs}$ ) purposely designed to guarantee the convergence. Beacons are exchanged periodically and can use the available SBCH super-frames illustrated in Fig. 2. In an environment where the closeness and the density of CNs make them prone to collision of transmitted beacon signals, there has been an intense research to reduce (or avoid) the collision of beacons [42-44].

Unlike conventional methods, here beacon collisions are induced and exploited for synchronization. Beacon signatures are designed to overlap one another but still enable the control by each device of the timing (TO) and carrier frequency (CFO) offsets errors to employ a distributed phase locked loop (D-PLL) algorithm [26]. The CNs cooperate with each other by making the beacons collide until a global network synchronization is reached without any external coordina- 
tion, or external TO/CFO reference. Distributed synchronization based on collision of pulse-like signatures were largely investigated for timing or frequency synchronization $[25,45,58]$. However, usage of pulse waveforms is not practical for WCN integration and this leaves the problem of joint TO and CFO synchronization as an open issue. Here, the beacon synchronization is designed based on chirp-like signatures arranged to solve the TO/CFO ambiguity and to enable joint TO-CFO synchronization [27]. The proposed periodic re-synchronization is designed to keep each node with carrier frequency and timing locked to the ensemble of carrier frequency and timings of the WCN network. Once the network has reached a consensus, the defined frames in Fig. 3-(a) can be kept aligned, giving to all nodes the same start of time-slot up to the propagation delay. Synchronization functions have been herein validated using a testing laboratory-scale hardware based on software-defined radio devices. In Sect. 5.4 we will discuss possible solutions for practical WCN system integration.

\subsection{Distributed Synchronization Algorithm}

Let us consider a dense WCN of $K$ connected and uncoordinated nodes statically deployed and mutually connected without any external synchronization reference. All CNs are deployed in a small geographic area, such as that the one proposed in Fig. 2: each signal transmitted can be received by almost all other nodes and propagation delay is negligible compared to inverse of its bandwidth. The network can be assumed to be strongly connected and each $\mathrm{CN}$ is half-duplex constrained (i.e., CN can either transmit or receive, but not both). Each node is equipped with independent oscillators that run autonomously and TO/CFO are locally re-synchronized to the ensemble of all the others by interactions. This retuning can be based on programmable frequency dividers of the local free-running oscillators. As discussed in Sect. 2, the time is discretized into frames (and superframes), while nodes are aware of the nominal frame period $T_{F}$ (this assumption can be relaxed as shown in [45]). In network systems, the node timings are nominally the same and oscillators of each (say $k$-th) node are affected by independent frequency fluctuations of local oscillators that change the frame-time $\tau_{k}[n]$ evaluated at $n$-th frame, and carrier angular frequency $\Omega_{k}(t)=\Omega_{o}+\omega_{k}(t)$ where $\Omega_{o}$ is the nominal carrier frequency. The transmitted synchronization signature $x(t)$ is the same for all CNs and it is free to collide (or superimpose) when confined inside the SBCH. All frames can be considered as mutually misaligned to one another, and delayed by the (absolute) time $\tau_{i}[n]$.

In half-duplex systems, each node is not-coordinated with the others and it chooses autonomously whether to transmit or receive. This generates a network 
topology that varies over time and connectivity can be modeled by a random topology without any guarantee to have balanced graphs at every duplex time $n$. The signal received by the $k$-th node over the $n$-th frame interval $T_{F}$ is the superposition of TO asynchronous transmitted signatures $x(t)$ by all the active neighboring nodes $\mathcal{N}_{k}[n]$ arbitrarily varying frame-to-frame:

$$
y_{k}(t \mid n)=\sum_{i \in \mathcal{N}_{k}[n]} h_{k i}(t) * x\left(t-\tau_{i}[n]\right) \exp \left(j \Omega_{i}(t) t\right)+w_{k}(t \mid n)
$$

Here signals are referred to the absolute time-reference just for the reasoning, but recall that this is not available to the nodes and this is replaced by the ensemble mean instead. $h_{k i}(t)$ is the channel response for the link $i \rightarrow k$ that accounts for multi-path fading and small node-to-node propagation delays, while $w_{k}(t \mid n)$ is the noise modeled as white Gaussian. The channel response is defined as $h_{k i}(t)=$ $g_{k i}(t) / r_{k i}^{\gamma}$, where $r_{k i}=r_{i k}$ is the distance between node $k$ and node $i$, and $g_{k i}(t)$ accounts for the fading process with $g_{k i}(t) \neq g_{i k}(t)$. The path-loss exponent $\gamma$ is typically in the range of 2 to 4.

The TOs and CFOs of the nodes belonging to $\mathcal{N}_{k}[n]$ are $\left\{\tau_{i}[n]\right\}_{i \in \mathcal{N}_{k}[n]}$ and $\left\{\omega_{i}(t)\right\}_{i \in \mathcal{N}_{k}[n]}$, respectively. These terms should be estimated by the $k$-th receiver in term of synchronization error with respect to the local reference: TO error is $\Delta \tau_{k i}[n]=\tau_{i}[n]-\tau_{k}[n]$ and CFO error is $\Delta \omega_{k}(t)=\omega_{i}(t)-\omega_{k}(t)$. Even if the synchronization method is consensus-based, the final solution does not converge to the average of the initial condition as in Sect. 4, due to the random topology [57], but, under mild conditions, the mutual TO and CFO synchronization is considered as achieved when $\left|\tau_{i}[n]-\tau_{k}[n]\right| \leq \sigma_{T O}$ and $\left|\omega_{i}(t)-\omega_{k}(t)\right| \leq \sigma_{C F O}$, for any pair $(i, k)$, where upper TO/CFO limits $\left(\sigma_{T O}, \sigma_{C F O}\right)$ depend on the datacommunication protocols. Usually, convergence conditions for TO and $\mathrm{CFO}$ are evaluated by the dispersion with respect to the mean $\mathrm{TO}$ or CFO over the entire cloud, and this is the mean square dispersion (MSD) metric detailed below.

The distributed synchronization algorithm is sequential as sketched in Fig. 5(a) where TO synchronization first reaches the steady state and this drives the convergence of the TO network. During frame alignment (TO acquisition, in Fig. 5-(b)), the CFO has a random behavior due to the large dispersion of TO not allowing a proper estimation of the CFO error. When TO is close enough to synchronization, the $\mathrm{CFO}$ correction takes place (CFO acquisition) by correcting the CFO impairments and allowing the fine synchronization of the network. To better illustrate the distributed performance, Fig. 5-(b) depicts the root MSD of TO and CFO towards synchronization. The scenario is the device-to-device (D2D) 


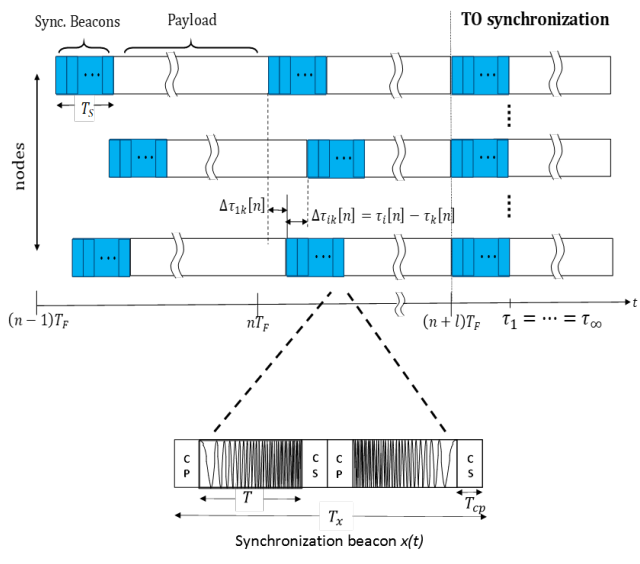

(a)

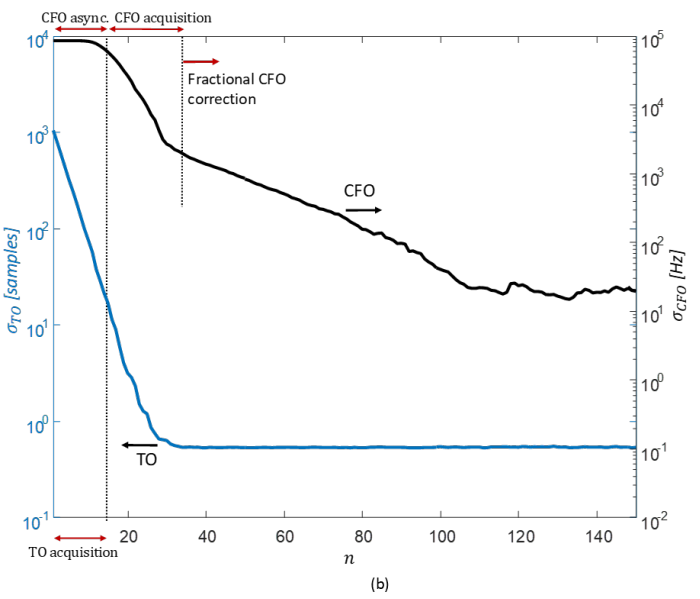

Figure 5: (a) TO synchronization evolution. All nodes transmit the same beacon $x(t)$ periodically during $T_{S}$ and on consecutive SBCH frames, i.e., under frame-period $T_{F}$. Beacons are allowed to collide among each other. At receiver node $k$, the relative TO error $\Delta \tau_{i k}[n]=\tau_{i}[n]-\tau_{k}[n]$ is the error from transmitted neighbor $\left(i \in \mathcal{N}_{k}\right)$ with respect to the local reference. The network reaches a synchronization stage when all the frames are time-aligned given the start of the frame. (b) Root mean-square deviation (MSD) of TO (left-axis) and CFO (right-axis) versus iteration for $K=50$ nodes mutually coupled and fully connected (all-to-all), and filter loop gain $\varepsilon_{T O}=\varepsilon_{C F O}=0.5$.

system [44] with carrier frequency of $2.4 \mathrm{GHz}$ and $N=64$ subcarriers (spacing $15 \mathrm{kHz}$ ). The maximum TO that can be tolerated is $\sigma_{T O}=1$ sample (sampling rate $1 / N$ ). Similarly, the maximum CFO that can be tolerated is $\sigma_{C F O}=100 \mathrm{~Hz}$. The numerical results show that the TO and CFO degradation of the distributed synchronization are lower than the maximum tolerated regardless of the density of the WCN: here $K=50$ nodes are considered. The distributed synchronization method fits the requirements of dense WCN, allowing scalability and reducing the algorithm complexity by using a single correlator filter at the receiver side.

\subsection{Distributed Phase Locked Loop (D-PLL)}

The D-PLL is an iterative control algorithm for synchronization that corrects the local TO/CFO of $k$-th node based on the estimation of the relative synchronization errors $\left(\Delta \tau_{k}[n]\right.$ and $\left.\Delta \omega_{k}(t)\right)$ with respect to the weighted average TO and CFO of the ensemble $\left|\mathcal{N}_{k}[n]\right|$ signatures. Fig. 5-(a) depicts the evolution of TO synchronization for the mutually misaligned frames.

Let $\theta_{k}[n]$ be the synchronization parameter that denotes here either CFO $\omega_{k}[n]$ or TO $\tau_{k}[n]$ at the $n$-th iteration, the consensus-based synchronization is based on the update (1) [26]. The method detailed herein estimates the relative TO 
or CFO $\Delta \theta_{k}[n]=\bar{\theta}_{k}[n]-\theta_{k}[n]$ from the superposition of the same synchronization signatures $x(t)$. The term $\bar{\theta}_{k}[n]=\sum_{i \in \mathcal{N}_{k}} \mathrm{w}_{k i}[n] \theta_{i}[n]$ is the weighted average of the ensemble synchronization parameters estimated from superimposed (colliding) synchronization signatures $x(t)$ of $\mathcal{N}_{k}$. The weighting term is $\mathrm{w}_{k i}[n]=\left|h_{k i}[n]\right|^{2} / \sum_{i \in \mathcal{N}_{k}[n]}\left|h_{k i}[n]\right|^{2}$ where $\mathrm{w}_{i i}[n]=0$. The CFO is periodically estimated during the synchronization period: it is $\bar{\omega}_{k}[n]=\left.\bar{\omega}_{k}(t)\right|_{t \in n T_{F}}$ constant within the frame. Both TOs and CFOs are periodically (every $T_{F}$ ) measured and corrected by comparing the local values with respect to the ensemble $\bar{\tau}_{k}[n]$ or $\bar{\omega}_{k}[n]$ to minimize the TO or CFO mismatch one another (Sect. 4).

In addition to the convergence speed expressed in terms of number of iterations, the key metric used for performance evaluation of the synchronization algorithm is the MSD of each synchronization parameter from the average: $\sigma_{\theta}^{2}[n]=$ $\frac{1}{K} \sum_{k=1}^{K} \mathbb{E}\left\{\left(\bar{\theta}[n]-\theta_{k}[n]\right)^{2}\right\}$. The term $\bar{\theta}[n]=\frac{1}{K} \sum_{k=1}^{K} \theta_{k}[n]$, and the expectation $\mathbb{E}\{\cdot\}$ are computed with respect to the random topology arising from duplexing. Stochastic perturbation on synchronization are caused by estimation error and oscillator's instability. These perturbations degrade the MSD at convergence, calling for an analysis of convergence for MSD that is monotonic decreasing $\sigma_{\theta}^{2}[n+1]<\sigma_{\theta}^{2}[n]$ and asymptotically $\lim _{n \rightarrow \infty} \sigma_{\theta}^{2}[n]=\sigma_{\theta}^{2}[\infty]$. The MSD $\sigma_{\theta}^{2}[\infty]$ can be minimized by choosing an optimum $\varepsilon$, even if the effective trade-off with convergence rate depends on the connectivity of the network.

\subsection{Collision-based Synchronization Parameters Estimation}

The estimation of the average TO $\bar{\tau}_{k}[n]=\mathcal{C}_{T O}\left\{y_{k}(t \mid n)\right\}$ and CFO $\bar{\omega}_{k}(t)=$ $\mathcal{C}_{C F O}\left\{y_{k}(t \mid n)\right\}$ from the superimposed beacons is framed as a joint estimation of time delay and frequency of superimposed signature copies $x(t)$ : notice that each signature is affected by different TOs and CFOs. Here, the severe noisy environment due to payload signals, if present, makes the TO/CFO estimates even more complex. The Maximum Likelihood Estimation (MLE) of TO/CFO for one node $\left(\left|\mathcal{N}_{k}\right|=1\right)$ is part of the values $(\tau, \omega)$ that maximizes the log-likelihood function [46]. For multiple beacons $\left(\left|\mathcal{N}_{k}\right|>1\right)$ as for distributed synchronization, the problem can be reduced to the selection of the TO and CFO centroids from the superposition of several delayed $x(t)$ as in (2).

The choice of the signature $x(t)$ is critical for synchronization. The linear frequency modulated pulse with sweep-rate $\bar{\mu}$ has good capabilities for time and frequency resolution [46]. The ambiguity function is smeared in the $(\tau, \omega)$ plane for joint TO/CFO estimation with benefit in term of noise reduction related to the signature length $T$. Since the bi-dimensional peak-search over the $(\tau, \omega)$ plane is 
too complex and time consuming for a real-time synchronization process, the $k$-th node can instead evaluate the matched filter output signal: $r(\tau)=\int y_{k}(t) x^{*}(t-$ $\tau) d t$. The received signal $y_{k}(t)$ is demodulated with the $k$-th local frequency to search now for the delay corresponding to the peak in $|r(t)|$. The TO/CFO ambiguity is removed by employing two sequential pulses shown in Fig. 5-(a) with sweep-rate $\pm \bar{\mu}$ so that the correlator receiver for the up-sweep $(q=1)$ and the down-sweep $(q=2)$ signatures are:

$$
r_{q}(\tau)=\int_{\tau-T / 2}^{\tau+T / 2} y_{k}(t) \exp \left(\mp j \bar{\mu} t^{2}\right) d t
$$

Assuming that the multi-path of the channel response is negligible as $h_{k i}(t)=$ $h_{k i} \times \delta(t)$, the matched filter is

$$
r_{q}(t)=\sum_{i \in \mathcal{N}_{k}} h_{k i} \mathcal{X}\left(t-\left(\tau_{i}-\tau_{k}\right), \pm\left(\omega_{i}-\omega_{k}\right)\right) \exp \left(j \Omega_{k i}\right)+\tilde{w}_{k}(t),
$$

with $\tilde{w}_{k}(t)=w_{k}(t) * \exp \left(j \bar{\mu} t^{2}\right) \operatorname{rect}(t / T)$, while

$$
\left|\mathcal{X}\left(\tau, \Delta \omega_{k}\right)\right|=\left(1-\frac{|\tau|}{T}\right)\left|\frac{\sin \left[\left(\Delta \omega_{k}-2 \bar{\mu} \tau\right)(T-|\tau|) / 2\right]}{\left(\Delta \omega_{k}-2 \bar{\mu} \tau\right)(T-|\tau|) / 2}\right|, \text { for }|\tau|<T
$$

is the $\operatorname{sinc}(\cdot)$ shaped ambiguity function with a main lobe of $2 \pi / \bar{\mu} T$ that becomes broader for large CFO $\Delta \omega_{k}$ errors [46]. The angular frequency is given by $\Omega_{k i}$ while the differential delays are $\tau_{k i}=\tau_{i}-\tau_{k} \pm\left(\omega_{i}-\omega_{k}\right) / 2 \bar{\mu}$ for the up/downsweep, respectively. In distributed synchronization, it is necessary to estimate the mean value of the TO and CFO of the neighboring nodes, and thus the barycentric delay value

$$
\hat{d}_{k, q}=\frac{\int t\left|r_{n}(t)\right|^{2} d t}{\int\left|r_{n}(t)\right|^{2} d t} \simeq \frac{\sum_{i \in \mathcal{N}_{k}}\left|h_{k i}\right|^{2} \tau_{k i}}{\sum_{i \in \mathcal{N}_{k}}\left|h_{k i}\right|^{2}}=\left(\hat{\bar{\tau}}_{k}-\tau_{k}\right) \pm \frac{\left(\hat{\bar{\omega}}_{k}-\omega_{k}\right)}{2 \bar{\mu}}
$$

is an estimator of the mean delay weighted by the amplitudes $\left|h_{k i}\right|^{2}$. The fine synchronization method refers to the estimation and correction of fractional CFO errors. This takes place after the network has reached a coarse synchronization when TO reaches a convergence and all frames are aligned up to a misalignment below the interval of $T$-samples. The fractional CFO is estimated based on conventional methods as in [27] based on fractional interpolation for consecutive signature sequences. 


\subsection{Practical Synchronization for Dense Network}

The Zadoff-Chu (ZC) sequences are used as practical chirps that can be implemented inside the WCN. These sequences are discrete-time linear frequency modulated waveforms with constant amplitude [47]. The good periodic correlation properties of the $\mathrm{ZC}$ sequences make them the preferred choice for both synchronization and channel estimation [48]. The ZC sequences can be arranged into a pair of up-sweep $(\bar{\mu}>0)$ and down-sweep $(\bar{\mu}<0)$ as shown in Fig. 5-(a) to resolve the TO/CFO ambiguity as for the continue-time chirp case.

In what follows, we consider the implementation of the distributed synchronization policy based on the baseband equivalent model of an OFDM system. The structure of the TO/CFO synchronization signature consists of two consecutive $\mathrm{ZC}$ sequences with the second one being the complex conjugate of the first one to get the up/down-sweeps. The discrete-time signature, see Fig. 5-(a), is composed of ZC sequences having $N$-samples length with a cyclic prefix (CP) and suffix (CS) each having $N_{c}$-samples length. $\mathrm{CP}$ and CS are introduced to account for selectivity of channel response by periodical shape:

$$
x[m]=\left\{\begin{array}{ll}
\exp \left(j \bar{\mu}\left(m-N_{c}\right)^{2}\right) & 0 \leq m \leq N_{T}-1 \\
\exp \left(-j \bar{\mu}\left(m-N_{c}-N_{T}\right)^{2}\right) & N_{T} \leq m \leq 2 N_{T}-1
\end{array},\right.
$$

for a support $N_{x}=2 N_{T}=2\left(N+2 N_{c}\right)$, where the sweep-rate for ZC is $\bar{\mu}=\frac{\pi}{N} \bar{u}$. The root index $\bar{u}$ is relatively prime to $N$, and it is $\bar{u}=1$ for convenience [27]. The $m$-th sample of the received signal is the superimposition of $\left|\mathcal{N}_{k}\right|$ transmitted waveforms affected by their relative TO and CFO errors:

$$
y_{k}[m]=\sum_{i \in \mathcal{N}_{k}} h_{k i} x\left[m-\tau_{k i}\right] \exp \left(j \omega_{k i} m\right)+w_{k}[m] .
$$

The estimation of coarse TO and CFO deviations is based on the timing metric that is obtained by two filters matched to the ZC sequence $x[\mathrm{~m}]$ and its conjugate replica $x^{*}[m]$ as in (4) for up/down sweep. At the acquisition stage, the TO/CFO are distant from each other, and coarse synchronization is based on the estimation of relative TO/CFO from the centroid of the matched filters for up/down sweeps (4) by:

$$
r_{k, q}[l]=\sum_{i \in \mathcal{N}_{k}} h_{k i} \mathcal{X}_{Z C}\left(l-\tau_{k i}, \pm \omega_{k i}\right)+\tilde{w}_{k, q}[l]
$$

where the ambiguity function $\mathcal{X}_{Z C}(\tau, \omega)$ is characterized by a periodic sinc $(\cdot)$ which contains the same (but opposite in sign) time shift that solve for TO/CFO 
the ambiguity similar to [49] for $\left|\mathcal{N}_{k}\right|=1$. The received signal $y_{k}[m]$ is filtered for each up- and down-sweep portion of signature $x[m]$. Each signal $r_{k, n}[l]$ has a different ambiguity function $\mathcal{X}_{Z C}(\tau, \omega)$. Furthermore, $r_{k, 1}[l]$ contains downsweep terms attenuated by ZC filtering, and similarly for $r_{k, 2}[l]$ with up-sweep terms. This mutual interference affects the up- and down- sweep barycenter estimator (6) with an increased interference proportional to $\left|\mathcal{N}_{k}\right|$. This mutual interference can be mitigated by increasing $N$, taking advance of the orthogonality properties of ZC sequences [47], even if in OFDM system the length $N$ is constrained by the number of sub-carriers.

Fine synchronization (i.e., tracking mode) for estimation and correction (1) for fractional CFO with $\varepsilon_{C F O^{F}}$ takes place after the network has reached a coarse TO synchronization and all frames are aligned up to a misalignment below the resolution of ZC ( $N$-samples). At this stage, small variations of CFO impair the performance and a fine correction must be applied. Thus, the fractional CFO is estimated based on a conventional MLE as the one proposed in [27] according to fractional interpolation for consecutive signature sequences.

Distributed synchronization on programmable GNU radios [50] shows good TO/CFO tracking and accuracy performance under a range of scenarios. The hardware demonstrator proves that uncoordinated $\mathrm{CNs}$ are likely to adapt their synchronization parameters to converge on a globally agreed timing and carrier frequency, without any coordination from the Host, and employing the same ZC sequences pair.

\section{Distributed Spectrum Sensing}

Self-learning of interference patterns is an essential task in the WCN. As exemplified in Fig. 6, the CNs have to detect the time-frequency (TF) interference patterns caused by the nodes (PN or Primary Nodes) of any pre-existing network to identify the unused portion of the spectrum and schedule the resources so as to avoid mutual interference [51]. Inferring the complete interference profile may not be feasible at each single $\mathrm{CN}$, due to the limited sensing range, the time-varying conditions associated with node mobility or the shadowing/fading effects. For instance, in Fig. 6 the TF resources of $\mathrm{PN}_{3}$ and $\mathrm{PN}_{4}$ are hidden in the data locally sensed by $\mathrm{CN}_{1}$. In this context, we propose to employ the consensus approach to virtually extend the perception capability of each $\mathrm{CN}$ by a distributed fusion of the data collected over the cloud network that relies on local interactions between neighboring nodes. 


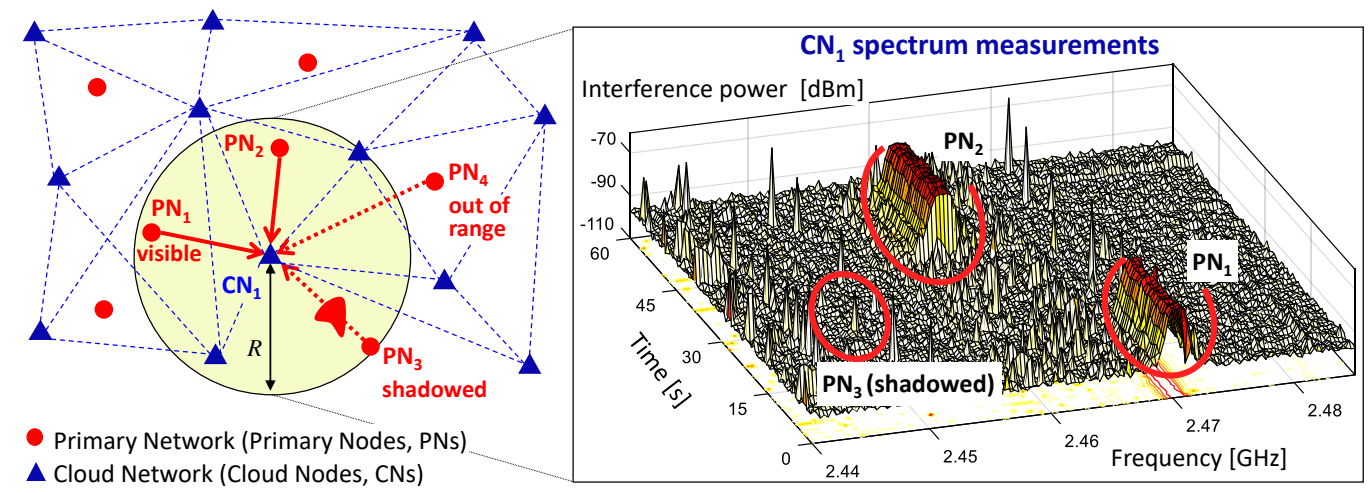

Figure 6: Cooperative spectrum sensing framework: $\mathrm{PN}_{1}$ and $\mathrm{PN}_{2}$ are sensed by $\mathrm{CN}_{1}$ as they both fall into its radio coverage area (yellow circle), whereas $\mathrm{PN}_{3}$ (shadowed) and $\mathrm{PN}_{4}$ (out of range) are hidden to $\mathrm{CN}_{1}$. In the right figure, an example of RSS measurements captured by $\mathrm{CN}_{1}$ is shown over the time-frequency grid. Cooperation among CNs through peer-to-peer links (blue dashed lines) allows detection of the overall PN interference pattern, regardless of the limited visibility at each $\mathrm{CN}$.

The cooperative sensing problem is analyzed in an experimental IoT scenario targeting the same $2.4 \mathrm{GHz}$ spectrum where the $\mathrm{WCN}$ is operating (see Sect. 2). Measurement campaigns have been carried out at Politecnico di Milano, with a WCN of $K=6$ CNs deployed in an area where three IEEE 802.15.4 (ZigBee) nodes and three WiFi devices are active in the same spectrum region. The spectrum locally sensed by the CNs is shown in Fig. 7-(a), which clearly highlights the poor visibility of the $\mathrm{PN}$ interferences at the $\mathrm{CNs}$, particularly at devices $\mathrm{CN}_{3}$ or $\mathrm{CN}_{5}$. The CNs engage in a distributed procedure to cooperatively identify the overall interference caused by the primary network (as shown in Fig. 7-(b)). The ZigBee PNs are assumed to perform a periodic transmission over predefined (but unknown to the $\mathrm{CNs}$ ) channels (TF resources) with center frequencies $\{2.45,2.46,2.47\} \mathrm{GHz}$ (standard-compliant with the channels $\{20,22,24\}$ ). With respect to the $\mathrm{CN}$ prototypes shown in Fig. 2, for the purpose of accurate spectrum sensing, the CNs are here connected with an external portable spectrum analyzer (embedded PC) by using a serial interface. This measures the received signal strength (RSS) over the band $2.4-2.485 \mathrm{GHz}$, with a configurable frequency step, here set to $\Delta f=333 \mathrm{kHz}$, resolution of $187.5 \mathrm{kHz}$ and sampling time (sweep time) $\Delta t=536 \mathrm{~ms}$. The RSS measurement set collected by the $k$ th $\mathrm{CN}$ is defined as $\mathcal{X}_{k}=\left\{x_{k}(t, f)\right\}$, where $(t, f)$ ranges over the TF resources of the spectrum. The RSS data-sets $\mathcal{X}_{k}$ are shown in Fig. 7-(a) for all the CNs $k=1, \ldots, K$. 


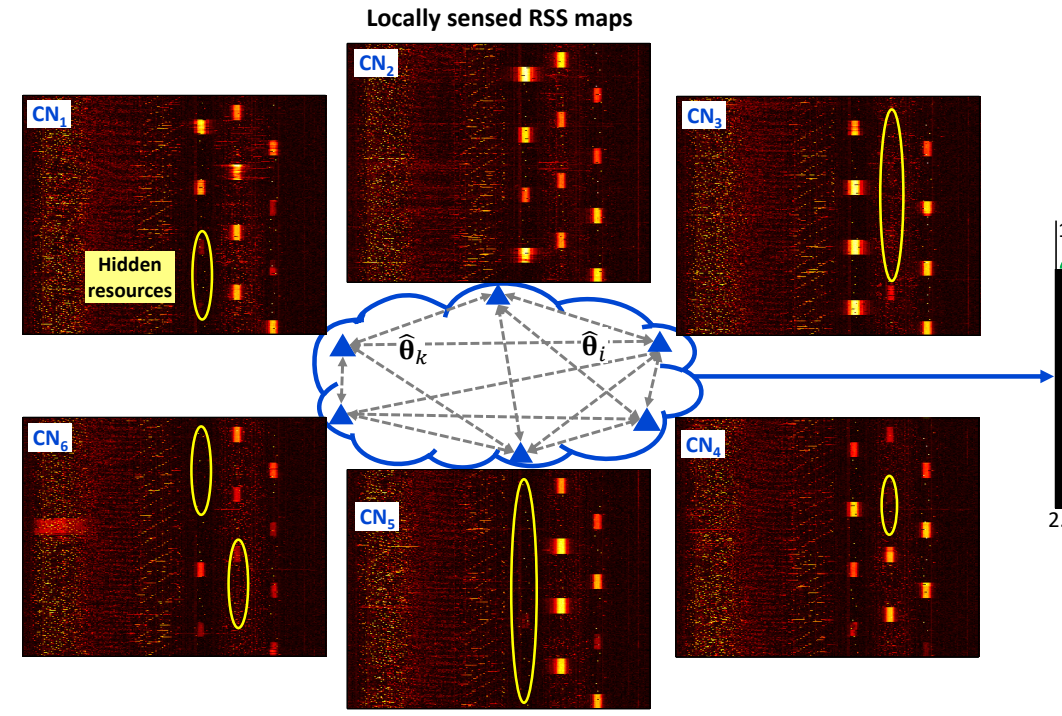

(a)

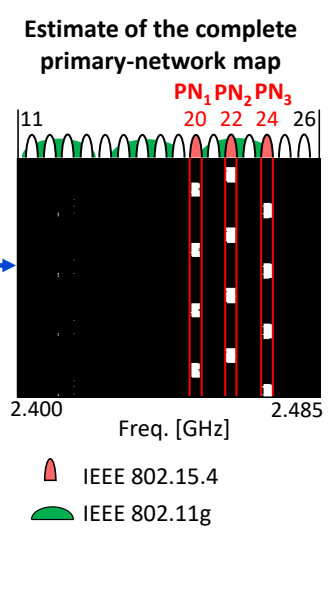

(b)

Figure 7: Distributed interference sensing in an IoT network, which consists of six IEEE 802.15.4 CNs and three IEEE 802.15.4 PNs performing periodic transmission tasks over the channels 20, 22 and 24. Three IEEE 802.11g devices are also interfering in the shared spectrum. (a) RSS measurements collected by CNs over time and frequency. (b) Time-frequency spectrum detection through the distributed sensing method.

For detection of the interference pattern, each RSS sample $x_{k}(t, f)$, according to the lognormal power model, is represented as a Gaussian random variable with parameters depending on the absence $\left(\mathcal{H}_{0}\right)$ or presence $\left(\mathcal{H}_{1}\right)$ of any PN signal in $(t, f)$. Under hypothesis $\mathcal{H}_{0}$, with probability $1-P$, the RSS $x_{k}(t, f) \sim \mathcal{N}\left(\mu_{0}, \sigma_{0}^{2}\right)$ models the background noise power with mean $\mu_{0}$ and variance $\sigma_{0}^{2}$. Under hypothesis $\mathcal{H}_{1}$, with probability $P, x_{k}(t, f) \sim \mathcal{N}\left(\mu_{1}, \sigma_{1}^{2}\right)$ models the PN signal power received at $\mathrm{CN} k$, with mean $\mu_{1}>\mu_{0}$ and variance $\sigma_{1}^{2}>\sigma_{0}^{2}$ (due to shadowing effects).

For known interference statistics, $\boldsymbol{\theta}=\left[\mu_{1}, \mu_{0}, \sigma_{1}^{2}, \sigma_{0}^{2}, P\right]^{\mathrm{T}}$, MAP (Maximum A-Posteriori) Bayesian detection of the PN signal is computed by comparing the RSS samples with the optimal threshold $S=S(\boldsymbol{\theta})$ resulting from standard likelihood-ratio test. However, the interference statistics $\theta$ are unknown at the cloud. Moreover, to reconstruct the complete interference pattern from the incomplete local data-sets, the WCN needs to aggregate all RSS data $\mathcal{X}=\left\{x_{k}(t, f)\right\}$ from all CNs $k=1, \ldots, K$ and TF resources $(t, f)$. To this aim, we propose the use of the consensus-based method [10] which enables the CNs to aggregate the 
information in a distributed manner, by the exchange of only partial belief metrics (using the available $\mathrm{SBCH}$ frames shown in Fig. 2) to minimize the inter-node signaling. The method combines the weighted-consensus algorithm (1) with an iterative decision directed (DD) procedure [52] for joint estimation of the PN statistics $\boldsymbol{\theta}$ and detection of the interfered TF resources associated with $\mathcal{H}_{1}$ (see details in [34]). Starting from an initial set $\hat{\boldsymbol{\theta}}^{(q)}$, at iteration $q=0,1,2, \ldots$, interference detection is performed inside each CN $k$ using the threshold $\hat{S}^{(q)}=S\left(\hat{\boldsymbol{\theta}}^{(q)}\right)$, and the local data $\mathcal{X}_{k}=\left\{x_{k}(t, f)\right\}$. Such data is partitioned into two subsets associated to the hypotheses $\mathcal{H}_{1}$ and $\mathcal{H}_{0}, \mathcal{X}_{\mathcal{H}_{1}, k}^{(q)}=\left\{x \in \mathcal{X}_{k}: x \geq \hat{S}^{(q)}\right\}$ and $\mathcal{X}_{\mathcal{H}_{0}, k}^{(q)}=\mathcal{X}_{k} \backslash \mathcal{X}_{\mathcal{H}_{1}, k}^{(q)}$, respectively. New interference parameters are obtained by computing the sample means $\left(\hat{\mu}_{1, k}^{(q+1)}, \hat{\mu}_{0, k}^{(q+1)}\right)$, variances $\left(\hat{\sigma}_{1, k}^{2(q+1)}, \hat{\sigma}_{0, k}^{2(q+1)}\right)$ and frequencies $\left(\hat{P}_{k}^{(q+1)}=\left|\mathcal{X}_{\mathcal{H}_{1}, k}^{(q)}\right| /\left|\mathcal{X}_{k}\right|\right)$ for the subsets $\mathcal{X}_{\mathcal{H}_{1}, k}^{(q)}, \mathcal{X}_{\mathcal{H}_{0}, k}^{(q)}$. Consensus iterations (1) are computed to aggregate the estimates at different $\mathrm{CNs}$ and to obtain the new set of PN statistics $\hat{\boldsymbol{\theta}}^{(q+1)}$ as virtually given by the fusion of $\left\{\mathcal{X}_{\mathcal{H}_{0}, k}^{(q)}, \mathcal{X}_{\mathcal{H}_{1}, k}^{(q)}\right\}_{k=1}^{K}$ over the whole cloud. The DD procedure is repeated till convergence when $\hat{P}_{k}^{(q)}=\hat{P}_{k}^{(q+1)}=\hat{P}_{k}^{(\infty)}$.

Fig. 7-(b) shows the binary mask obtained by the distributed detection of the complete TF interference patterns caused by the three IEEE 802.15.4 PN devices. It can be shown that the distributed method based on weighted-consensus is able to reach the centralized detection performance [28].

\section{Distributed Radio Sensing for Localization and Vision Reconstruction}

Localization and vision technologies are expected to play a key role in next generation of cyber-physical systems. In the specific field of IIoT applications, the tight integration of physical (e.g., robots, manipulators) and software components (e.g., control, monitoring) is made more complex by the contact-free interactions between machines, robots and human workers. Radio sensing technologies exploit RF signals exchanged between the CNs to sense, detect and monitor alterations of the propagation environment that are induced by people or objects (e.g., targets) moving inside the WCN coverage area. These methods in turn allow device-free (also known as tag-free or passive) human body motion recognition, by relying solely on acquisition and processing of the electromagnetic (EM) field propagated for the $\mathrm{CN}$ connectivity. Body-induced alterations of the EM field, that covers the monitored area, are measured and processed in real-time by the CNs to extract information about the subject (e.g., presence, position, movements, or 


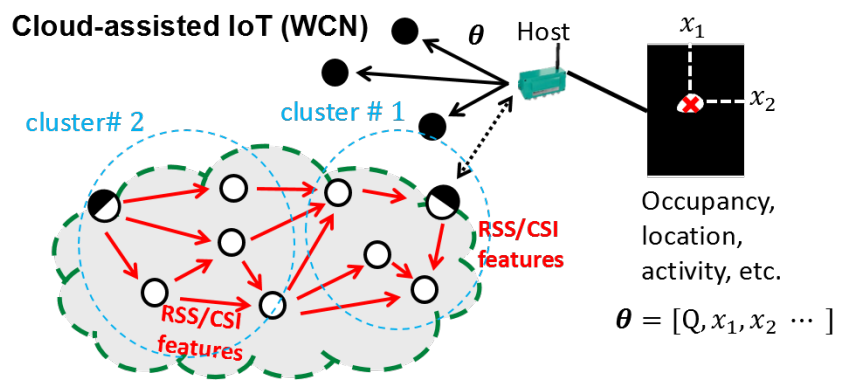

(a)

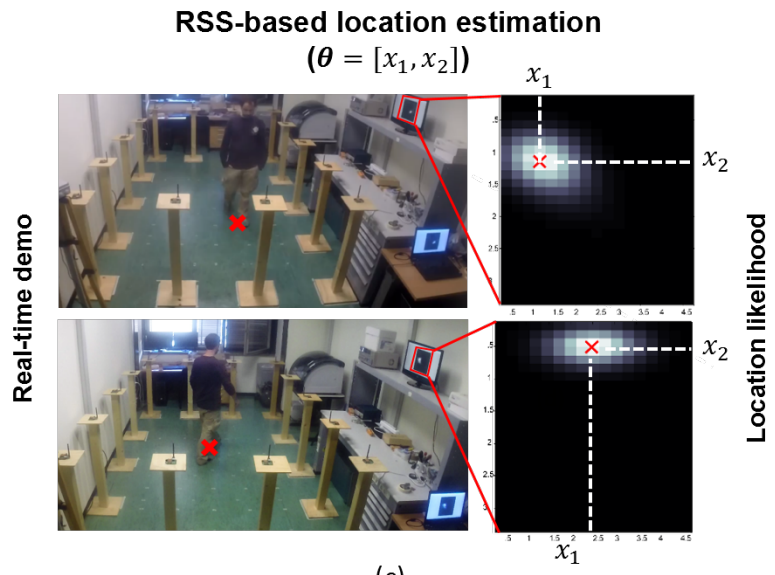

RSS-based occupancy estimation $(\theta=Q)$
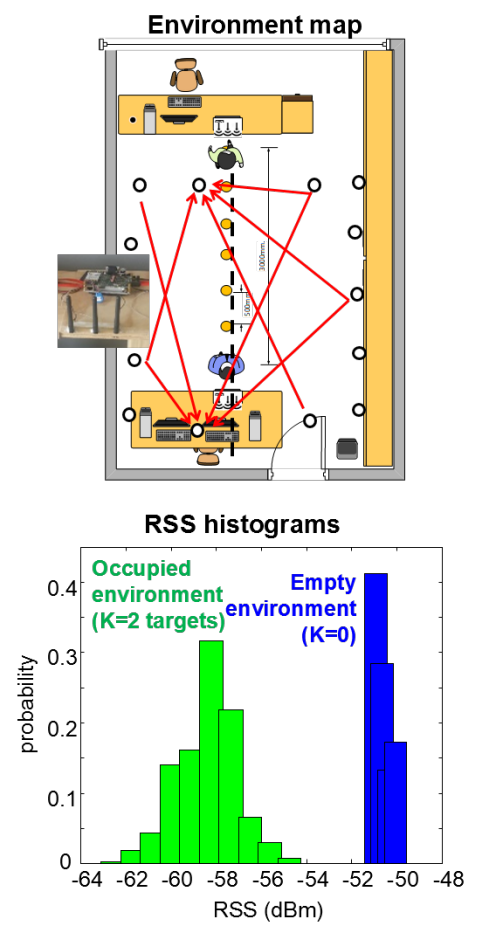

(b)

Figure 8: (a) Cloud-assisted distributed estimation of user location or occupancy based on RSS features exchanged among CNs, (b) device-free occupancy estimation using a network of $14 \mathrm{CNs}$ (top) and RSS histograms from individual CNs (bottom), (c) device-free location estimation using a network of $14 \mathrm{CNs}$ (left) to compute the likelihood maps (right).

activities) [12] [53] or to compute an image of the environment that originated the EM perturbation. These techniques have the advantage of not requiring any wearable device (e.g., radio tag), which, in most industrial environments, is largely unfit; in addition, this technology can be used also in presence of fumes, vapors and occluding materials. Last but not least, these methods are privacy preserving as they extract only geometrical information about the target from the RF signals (i.e., position, size, posture).

Device-free positioning is applied in industrial workplace to track and protect operators, and to support safety, particularly in shared human-machine workplace [54]. As shown in Fig. 8-(a), each CN collects channel quality information (CQI) from the physical layer, such as the channel state information (CSI), or upper 
layers, such as the RSS, and sends this information to the CA nodes. Considering that the $\mathrm{CN}$ prototype (Sect. 2) does not support low-level CSI monitoring functions, we focus here on distributed processing of RSS measurements that are exchanged over the SBCH channels. The RSS power level (expressed in $\mathrm{dBm}$ ), that is measured by a $\mathrm{CN}$, can be expressed as $P(\boldsymbol{\theta})=P_{0}-A_{T}(\boldsymbol{\theta})+w \cdot P_{0}$ is the deterministic path-loss term that depends only on the geometry and the propagation characteristics of the empty scenarios (i.e., the environment without any target inside). The Gaussian random variable $w$ includes the lognormal terms due to both multi-path and measurement disturbances. The extra attenuation term $A_{T}(\boldsymbol{\theta})$ is due to the body-induced effects with respect to the empty scenario. According to [55], $A_{T}(\boldsymbol{\theta})$ can be modeled in terms of monitored parameters $\boldsymbol{\theta}$ such as the target position, the geometry and the small movements. Cloud devices thus act as virtual sensors, as they communicate with neighbors to fuse, process CQI data locally and perform low-level decisions on the sensed parameters $\boldsymbol{\theta}$. For example, occupancy detection is a low level sensing task where $\boldsymbol{\theta}$ represents the spatial density of parameters that is inferred by processing the RSS measured over different sub-channels and links. CNs independently extract and evaluate features from RSS data to highlight any anomalous alteration of the EM field as possibly induced by body presence. These features can be defined in terms of extra attenuation, mean, likelihood, and spatial/frequency correlations of the received RF signals [56]. Detected features indicating potential anomalies in the RSS field are then shared with neighbor CNs, via MAC-layer cooperative transmission functions (Sect. 3). Finally, a consensus algorithm (Sect. 4) is used to reach a decision about body presence. Information about body motions can be used by the cloud for high-level sensing tasks such as device-free positioning (where $\boldsymbol{\theta}$ represents the targets' locations), target counting (with $\boldsymbol{\theta}$ representing the number of targets) or activity recognition (with $\boldsymbol{\theta}$ denoting the type of activity).

Fig. 8 shows the experimental layout for occupancy detection in an indoor environment with two targets. In Fig. 8-(b), the RSS histograms highlight the bodyinduced perturbations of the radio signal strength observed by a $\mathrm{CN}$, compared with the empty environment, where no target is inside the monitored area. Targets are moving along the line-of-sight (LOS) path. As shown in this figure, RSS values are sensitive to the presence of the subject in the surroundings of the CNs, thus making detection of the occupied environment possible through RSS data inspection. In Fig. 8-(c), the localization of the subject is obtained by distributed fusion of the RSS features computed by 14 CNs. Localization can be implemented based on the consensus approach. Consensus is here based on distributed fusion of local log-likelihood information obtained by individual CNs. The location likelihood 
map obtained by the consensus procedure is plotted in Fig. 8-(c) on the right, where the target location $\boldsymbol{\theta}=\left[x_{1}, x_{2}\right]^{\mathrm{T}}$ is estimated according to the maximum likelihood criterion [54].

\section{Concluding Remarks}

In this paper, we first introduced a WCN platform, that can lease advanced communication and sensing services to off-the-shelf IoT wireless devices via a dense network of self-organizing wireless nodes. The WCN paradigm is underpinned by distributed signal processing and offers the potential for improved reliability, connectivity and latency compared with current IoT solutions. Distributed synchronization is the enabling technology for implementation of PHY layer cooperative communication envisioned in future $5 \mathrm{G}$ standardization. An experimental case study inside a pilot industrial plant considered the problem of critical data publishing (including monitoring and fast request-response datagram exchange). An overview of distributed information processing methods was then provided with focus on weighted consensus-based approaches. These algorithms have been exploited to solve a number of specific inference problems, such as the estimation of communication parameters (i.e., timing and carrier frequency offset), interference sensing and device-free positioning.

The advantages of the wireless cloud networking solutions illustrated in this paper are shown to be relevant for dense deployments characterized by poor radio signal quality (due to harsh propagation conditions or severe building blockage). The use of the cooperative multi-hop transmission chain strategy provides a sufficiently high level of immunity to multi-path fading and interference. In addition, the proposed distributed synchronization tool is expected to enable an efficient scheduled access and determinism of communication. This is particularly crucial in latency-critical real-time control (including process control and safety appli-

cations). Compared to current industry-standard networks, the WCN allows to scale down the maximum latency from 6 up to 10 times. Future work will consider the applicability of the proposed platform in emerging high-frequency radio networks, ranging from the $60 \mathrm{GHz}$ to the sub-THz bands $(100-150 \mathrm{GHz})$.

\section{Bibliography}

\section{References}

[1] O. Vermesan and P. Friess, Internet of Things - from Research and Innovation to Market Deployment. River Publishers Aalborg, 2014. 
[2] M. R. Palattella, M. Dohler, A. Grieco, G. Rizzo, J. Torsner, T. Engerl, L. Ladid, "Internet of Things in the 5G era: enablers, architecture, and business models," IEEE J. on Selected Areas in Communications, vol. 34, no. 3, Mar. 2016.

[3] L. D. Xu, W. He and S. Li, "Internet of Things in Industries: a survey," IEEE Trans. in Industrial Informatics, vol. 10, no. 4, pp. 2233-2243, Nov. 2014.

[4] T. Watteyne et al., "Industrial Wireless IP-Based Cyber-Physical Systems," Proc. of the IEEE, vol. 104, no. 5, pp. 1025-1038, May 2016.

[5] T. Rault, A. Bouabdallah, Y. Challal, "Energy efficiency in wireless sensor networks: a top-down survey," Computer Networks, vol. 67, no. 4, pp. 104122, Jul. 2014, ISSN 1389-1286.

[6] P. Suriyachai, U. Roedig, A. Scott, "A survey of MAC protocols for missioncritical applications in wireless sensor networks," IEEE Communications Surveys \& Tutorials, vol. 14, no. 2, 2012.

[7] J. Sỳkora and H. Mark, "Dense Cooperative Wireless Cloud Network (DIWINE)," Proc of the Future Network and Mobile Summit (FuNeMS), pp. 3-5, 2013.

[8] H. Mark, P.H. Lin, J. Sỳkora, K. Ramantas, D. Boixade, S. Galimberti, U. Spagnolini, M. Nicoli, D.E. Halls, and S. Savazzi, "Enhancing wireless communications," Pan European Networks: Science \& Technology, No. 17, pp. 106-108, Dec. 2015.

[9] S. Savazzi, V. Rampa, U. Spagnolini, 'Wireless Cloud Networks for the Factory of Things: Connectivity Modeling and Layout Design," IEEE Internet of Things Journal, vol. 1, no. 2, Apr. 2014.

[10] L. Ascorti, S. Savazzi, G. Soatti, M. Nicoli, E. Sisinni, S. Galimberti, “A wireless cloud network platform for industrial process automation: Critical data publishing and distributed sensing," IEEE Trans. on Instrumentation and Measurement, vol. 66, no. 4, pp. 592-603, Apr. 2017.

[11] H. Kurunathan, R. Severino, A. Koubaa, E. Tovar, "IEEE 802.15.4e in a Nutshell: Survey and Performance Evaluation," IEEE Communications Surveys \& Tutorials, doi: 10.1109/COMST.2018.2800898, vol. 20, no. 3, pp. 19892010, thirdquarter 2018. 
[12] S. Savazzi, S. Sigg, M. Nicoli, V. Rampa, S. Kianoush and U. Spagnolini, "Device-Free Radio Vision for Assisted Living: Leveraging wireless channel quality information for human sensing," IEEE Signal Processing Magazine, vol. 33, no. 2, pp. 45-58, March 2016.

[13] D. I. Shuman, S. K. Narang, P. Frossard, A. Ortega, P. Vandergheynst, "The emerging field of signal processing on graphs: Extending high-dimensional data analysis to networks and other irregular domains," IEEE Signal Processing Magazine, vol. 30, no. 3, pp. 83-98, May 2013.

[14] A. Sandryhaila and J. M. F. Moura, "Big data analysis with signal processing on graphs: Representation and processing of massive data sets with irregular structure," IEEE Signal Processing Magazine, vol. 31, no. 5, pp. 80-90, Sep. 2014.

[15] M. Tsitsvero, S. Barbarossa, and P. Di Lorenzo, "Signals on graphs: Uncertainty principle and sampling," IEEE Trans. on Signal Processing, vol. 64, no. 18, pp. 4845-4860, Sep. 2016.

[16] I. Khan, F. Belqasmi, R. Glitho, N. Crespi, M. Morrow and P. Polakos, "Wireless sensor network virtualization: a survey," IEEE Communications Surveys \& Tutorials, vol. 18, no. 1, pp. 553-576, 2016.

[17] S. Ma, Y. Yang and H. Sharif, "Distributed MIMO technologies in cooperative wireless networks," IEEE Communications Magazine, vol. 49, no. 5, pp. 78-82, May 2011.

[18] X. Tao, X. Xu and Q. Cui, "An overview of cooperative communications," IEEE Communications Magazine, vol. 50, no. 6, pp. 65-71, Jun. 2012.

[19] S. Savazzi, "Wireless virtual multiple antenna networks for critical process control: protocol design and experiments," International J. of Distributed Sensor Networks, vol. 2013, Article ID 973621, 15 pages, 2013.doi:10.1155/2013/973621.

[20] P. Castiglione, S. Savazzi, M. Nicoli, T. Zemen, "Partner Selection in Indoorto-Outdoor Cooperative Networks: An Experimental Study," IEEE Journal on Selected Areas in Communications, vol. 31, no. 8, pp. 1559-1571, August 2013. 
[21] N. Marchenko, T. Andre, G. Brandner, W. Masood, C. Bettstetter, "An experimental study of selective cooperative relaying in industrial wireless sensor networks," IEEE Trans. on Industrial Informatics, vol. 10, no. 3, Aug. 2014.

[22] P. Braca, S. Marano, and V. Matta, "Enforcing Consensus While Monitoring the Environment in Wireless Sensor Networks," IEEE Trans. on Signal Processing, vol. 56, no. 7, pp. 3375-3380, Jul. 2008.

[23] G. Soatti, M. Nicoli, N. Garcia, B. Denis, R. Raulefs, H. Wymeersch, "Implicit Cooperative Positioning in Vehicular Networks," IEEE Trans. on Intelligent Transportation Systems, doi: 10.1109/TITS.2018.2794405, vol. 19, no. 12, pp. 3964-3980, Dec. 2018.

[24] A. Pascale, M. Nicoli, and U. Spagnolini, "Cooperative Bayesian Estimation of Vehicular Traffic in Large-Scale Networks," IEEE Trans. on Intelligent Transportation Systems, vol. 15, no. 5, pp. 2074-2088, Oct. 2014.

[25] Y.-W. Hong and A. Scaglione, "A Scalable Synchronization Protocol for Large Scale Sensor Networks and its Applications," IEEE J. on Selected Areas in Communications, vol. 23, no. 5, pp. 1085-1099, 2005.

[26] O. Simeone, U. Spagnolini, Y. Bar-Ness, and S. H. Strogatz, "Distributed Synchronization in Wireless Networks," IEEE Signal Processing Magazine, vol. 25, no. 5, pp. 81-97, 2008.

[27] M. A. Alvarez and U. Spagnolini, "Distributed Time and Carrier Frequency Synchronization for Dense Wireless Networks," IEEE Transactions on Signal and Information Processing over Networks, vol. 4, no. 4, pp. 683-696, Dec. 2018.

[28] G. Soatti, M. Nicoli, S. Savazzi, U. Spagnolini, "Consensus-based Algorithms for Distributed Network-State Estimation and Localization," IEEE Trans. on Signal and Information Processing over Networks, vol. 3, no. 2, pp. 430-444, Jun. 2017.

[29] M. Z. Win, A. Conti, S. Mazuelas, Y. Shen, W. M. Gifford, D. Dardari, and M. Chiani, "Network Localization and Navigation via Cooperation," IEEE Communications Magazine, vol. 49, no. 5, pp. 56-62, May 2011.

[30] G. C. Calafiore, L. Carlone, and M. Wei, "A Distributed Technique for Localization of Agent Formations from Relative Range Measurements," IEEE 
Trans. on Systems, Man, and Cybernetics-Part A: Systems and Humans, vol. 42, no. 5, pp. 1065-1076, 2012.

[31] U. A. Khan, S. Kar, and J. M. Moura, "Distributed Sensor Localization in Random Environments using Minimal Number of Anchor Nodes," IEEE Trans. on Signal Processing, vol. 57, no. 5, pp. 2000-2016, 2009.

[32] A. Ghasemi and E. S. Sousa, "Collaborative Spectrum Sensing for Opportunistic Access in Fading Environments," in First IEEE International Symposium on New Frontiers in Dynamic Spectrum Access Networks (DySPAN), 2005, pp. 131-136.

[33] P. Di Lorenzo, S. Barbarossa, and A. H. Sayed, "Distributed Spectrum Estimation for Small Cell Networks based on Sparse Diffusion Adaptation," IEEE Signal Processing Letters, vol. 20, no. 12, pp. 1261-1265, 2013.

[34] G. Soatti, M. Nicoli, S. Savazzi, and U. Spagnolini, "Distributed Sensing of Interference Pattern in Dense Cooperative Wireless Networks," IEEE International Conference on Communications (ICC), London, 8-12 June 2015, pp. 961-966.

[35] P. Di Lorenzo, S. Barbarossa, and A. H. Sayed, "Bio-Inspired Decentralized Radio Access based on Swarming Mechanisms over Adaptive Networks," IEEE Trans. on Signal Processing, vol. 61, no. 12, pp. 3183-3197, 2013.

[36] F. S. Cattivelli and A. H. Sayed, "Analysis of Spatial and Incremental LMS Processing for Distributed Estimation," IEEE Trans. on Signal Processing, vol. 59, no. 4, pp. 1465-1480, Apr. 2011.

[37] A. H. Sayed, S. Y. Tu, J. Chen, X. Zhao, Z. J. Towfic, "Diffusion Strategies for Adaptation and Learning over Networks: An Examination of Distributed Strategies and Network Behavior," IEEE Signal Processing Magazine, vol. 30, no. 3, pp. 155-171, May 2013.

[38] A. Simonetto and G. Leus, "Distributed Maximum Likelihood Sensor Network Localization," IEEE Trans. on Signal Processing, vol. 62, no. 6, pp. 1424-1437, Mar. 2014.

[39] H. Wymeersch, J. Lien, and M. Z. Win, "Cooperative Localization in Wireless Networks," Proceedings of the IEEE, vol. 97, no. 2, pp. 427-450, Feb. 2009. 
[40] R. Olfati-Saber, J.A. Fax, and R.M. Murray, "Consensus and Cooperation in Networked Multi-Agent Systems," Proceedings of the IEEE, vol. 95, no. 1, pp. 215-233, Jan. 2007.

[41] I. D. Schizas, A. Ribeiro, and G.B. Giannakis, "Consensus in Ad Hoc WSNs with Noisy Links. Part I: Distributed Estimation of Deterministic Signals," IEEE Trans. on Signal Processing, vol. 56, no. 1, pp. 350-364, Jan. 2008.

[42] Y. Xu, and A. Helal, "Scalable Cloud-Sensor Architecture for the Internet of Things," IEEE J. Internet of Things, vol. 3, no. 3, pp.285-298, Jun. 2016.

[43] K. J. Zou, and K. W. Yang, "Network Synchronization for Dense Small Cell Networks," IEEE Wireless Communications, vol. 22, no. 2, pp.108-117, Apr. 2015.

[44] N. Abedini and S. Tavildar and J. Li and T. Richardson, "Distributed Synchronization for Device-to-Device Communications in an LTE Network," IEEE Trans. Wireless Communications, vol. 15, pp.1547-1561, Feb. 2016.

[45] O. Simeone and U. Spagnolini, "Distributed Time Synchronization in Wireless Sensor Networks with Coupled Discrete-Time Oscillators," EURASIP J. Wireless Communications and Networking, vol. 2, pp. 690-694, Sep. 2007.

[46] H. L. V. Trees, "Detection, Estimation, and Modulation Theory, Part III: Radar Sonar Signal Processing and Gaussian Signals in Noise," John Wiley \& Sons, INC., 2001.

[47] J. W. Kang, Y. Whang, B. H. Ko, and K. S. Kim, "Generalized CrossCorrelation Properties of Chu Sequences," IEEE Trans. Information Theory, vol. 58, no. 1, pp. 438-444, Jan. 2012.

[48] "Technical specification group radio access network; Physical layer aspects for evolved universal terrestrial radio access (UTRA) (Release 7)," Cedex, Tech. Rep. 3GPP TR 25.814, V7.1.0, Sep. 2006.

[49] M. Gul, X. Ma, and S. Lee, "Timing and Frequency Synchronization for OFDM Downlink Transmissions Using Zadoff-Chu Sequences," IEEE Trans. Wireless Communications, vol. 14, no. 3, pp. 1716-1729, Mar. 2015. 
[50] M. A. Alvarez, W. Thompson and U. Spagnolini, "Distributed Time and Frequency Synchronization: USRP Hardware Implementation," IEEE International Conference on Communication Workshop (ICCW), pp. 2157-2162, Jun. 2015.

[51] S. Mishra, A. Sahai, R. Brodersen, "Cooperative sensing among cognitive radios," IEEE International Conference on Communications (ICC), pp. 1658-1663, Jun. 2006.

[52] P. N. Tan, M. Steinbach, and V. Kumar, Introduction to data mining. Pearson Education, 2006.

[53] S. Kianoush, S. Savazzi, and V. Rampa, "Leveraging MIMO-OFDM radio signals for device-free occupancy inference: system design and experiments, "EURASIP Journal on Advances in Signal Processing, Vol. 44, 2018.

[54] S. Kianoush, S. Savazzi, F. Vicentini, V. Rampa, and M. Giussani, "DeviceFree RF Human Body Fall Detection and Localization in Industrial Workplaces, ” IEEE Internet of Things Journal, vol. 4, no. 2, pp. 351-362, 2017.

[55] V. Rampa, G. G. Gentili, S. Savazzi, and M. D'Amico, "EM Models for Passive Body Occupancy Inference," IEEE Antennas and Wireless Propagation Letters, vol. 16, pp. 2517-2520, 2017.

[56] S. Kianoush, M. Raja, S. Savazzi and S. Sigg, "A Cloud-IoT Platform for Passive Radio Sensing: Challenges and Application Case Studies," IEEE Internet of Things Journal, vol. 5, no. 5, pp. 3624-3636, Oct. 2018.

[57] F. Fagnani, and S. Zampieri, "Randomized Consensus Algorithms over Large Scale Networks," Information Theory and Applications Workshop, pp. 50-159, Jan. 2007.

[58] N. Varanese, U. Spagnolini, and Y. Bar-Ness, "Distributed FrequencyLocked Loops for Wireless Networks," IEEE Transactions on Communications, vol. 59, no. 12, pp. 3440-3451, December 2011. 